\title{
Monitoring of the pulsation cycle in a jig using a radiometric density meter
}

\author{
Stanislaw Cierpisz ${ }^{1}$, and Jaroslaw Joostberens ${ }^{2, a}$ \\ ${ }^{1}$ Institute of Innovative Technologies EMAG, Katowice, Poland \\ ${ }^{2}$ Silesian University of Technology, Gliwice, Poland
}

\begin{abstract}
Application of a radiometric density meter to monitor changes in coal bed density during a pulsation cycle in a jig has been presented. The signal from the radiation detector has been filtered in an adaptive filter to react properly to rapid changes in density. The radiometric density meter has been used to measure density of a coal layer $3 \mathrm{~cm}$ thick and $30 \mathrm{~cm}$ wide. The distribution of the material density along the bed height has been measured during field tests performed on the industrial jig. Changes in density have shown the process of material loosening and its compression during each cycle of pulsations.
\end{abstract}

\section{Introduction}

Raw coal is often beneficiated by gravity separation processes where coal grains are stratified according to their density in a pulsating coal/water medium in jigs (Fig. 1). These processes have been discussed in [1-3]. During subsequent water pulsations induced by opening and closing air valves, coal stratification occurs as grains of various density move upwards and downwards with different velocities. Grains of low density (low ash content) migrate to upper layers with grains of high density (high ash content) moving down to lower layers.

Stratification of grains due to their density is not ideal. Their upward and downward movement also depends on their diameters, shape and the way in which the material loosens within each pulsation cycle. Simulation models of fine coal segregation and stratification processes in jigs have been discussed elsewhere [4-5]. The material travels horizontally on a screen deck along the jig compartment with the flow of water. At the end of the jig compartment the stratified material is split to upper (concentrate) and bottom (refuse or middlings) products. This process is controlled by the refuse discharge system influencing the tonnage of the bottom product.

The most popular refuse discharge control systems in jigs use a metal float to monitor the thickness of the heavy fraction in a jig compartment. Although the float is the main sensor, its operation as a measuring unit has not been adequately studied so far. The control system stabilizes the position of the float at the chosen desired value adjusting the tonnage of the bottom product and in this way stabilizing, to some extent, the separation density. The separation density is the density of the coal layer reporting in half to the upper product and in half to the refuse. Monitoring and control

${ }^{a}$ Corresponding author: Jaroslaw.Joostberens@polsl.pl 
systems applied commercially at present in jigs are limited to control operation of air valves in order to stabilize the shape of the bed pulsation and to control the tonnage of the discharged bottom product.

Laboratory and industrial tests show fluctuations of the position of a float in relation to the position of the measured layer. These fluctuations appear as a result of changes in the feed tonnage and the feed washability characteristics. The changes in the degree of material loosening produce changes in velocity of float during upward and downward movements in subsequent pulsation cycles. The investigations showed the fluctuations of the separation density of the order of $0.05 \mathrm{~g} / \mathrm{cm}^{3}$ and sometimes even more [6]. This can result both in fluctuations of tonnages and ash content in products, and in coal losses in refuse. Raw fine coal washing in jigs can be more efficient when a float in a refuse discharge control system is replaced by a radiometric density meter.

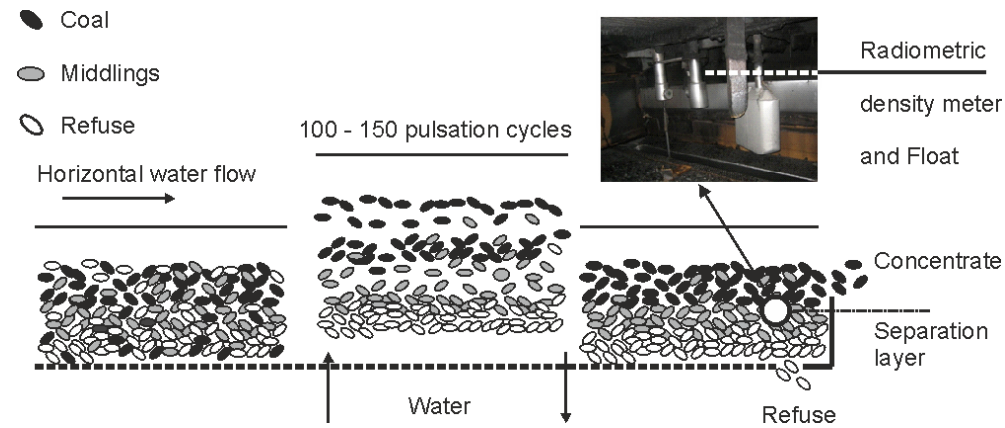

Figure 1. Illustration of a coal separation process in a jig.

First application of a radiometric density meter to control a jig operation (imperfection reduced from 0.15 to 0.08 ) was reported elsewhere [7]. Much more advanced system with a radiometric density meter, applied to control a jig operation (JigScan), was described elsewhere [8]. A radiometric density meter, applied to monitor the density of a jig's bed at different levels, appeared to be a good tool used for determination of both static and dynamic characteristics of a jig [9]. Results of these investigations have been presented in this paper.

\section{Radiometric density meter}

The radiometric density meter used in the experimental system consisted of a waterproof container for the radiation source $\left({ }^{137} \mathrm{Cs}\right)$ and the scintillation counter as the gamma radiation detector - Figure 2 . The radiation beam was collimated (ca. $3 \mathrm{~cm}$ diameter) and absorbed by the $30 \mathrm{~cm}$ long layer of the pulsating media.

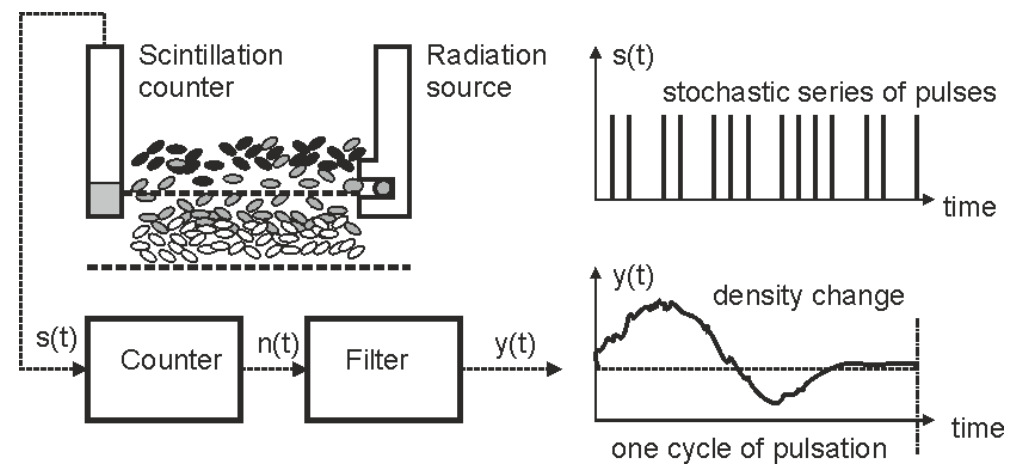

Figure 2. Radiation density meter. 
The output signal from the radiation detector $s(t)$ is a nonstationary signal of series of pulses of Poisson distribution presented by equation (1):

$$
p(n, k)=\frac{n(t)^{k} \cdot e^{-n(t)}}{k !}
$$

where:

$p(n, k)$ - probability of counting $k$ pulses in the time interval $\left(t, t+t_{s}\right)$,

$n(t)$ - mean intensity of pulses in time $t$.

The density of the bed was determined according to the absorption law (2):

$$
\rho=1-\frac{\ln \left(\frac{I_{x}}{I_{w}}\right)}{\mu d}=1-\frac{\ln \left(\frac{N x}{N_{w}}\right)}{\mu d}
$$

where:

$I_{0}$ - incident radiation intensity,

$I_{w}$ - radiation intensity registered by the

detector,

$N_{w}$ - number of pulses counted by the detector

for water,

$I_{x}$ - radiation intensity detected by the detector

$N_{x}$ - number of pulses counted by the detector for media (coal/water),

$d$ - the thickness of the absorbent, $\mathrm{cm}$,

$\mu$ - mass coefficient of gamma absorption, $\mathrm{cm}^{2} / \mathrm{g}$, $\rho$-density of media, $\mathrm{g} / \mathrm{cm}^{3}$.

for media (coal/water),

For small changes in the density, Equation (2) can be transformed and linearized:

$$
n(t)=n_{0}-k_{n} \cdot \rho(t)
$$

where:

$n_{0} \quad$ intensity of pulses for the reference density (water),

$k_{n} \quad-$ coefficient.

The counter output signal is converted to the form $u$ in discrete time moments $i$ using the equation:

$$
u[i]=\frac{1}{k_{n}} \cdot\left(n_{0}-\frac{k[i]}{t_{s}}\right)
$$

where:

$k[i]$ - number of pulses from the detector counted by counter during time $t+t_{s}$, $i=t / t_{s}$.

The signal $u[i]$ consists of the measured value of the density and the stochastic error $e[i]$ :

$$
u[i]=\rho[i]+e[i] .
$$

The output signal from the filter $y[i]$ is given by equation:

$$
y[i]=u[i]-\varepsilon[i] .
$$

The output signal $u[i]$ is a stochastic signal which should be filtered as it consists of the useful signal $\rho(t)$ and a noise. One of the methods of predictive filtration of $u[i]$ is presented in details in [10]. The predictive filter is described by equation: 


$$
\rho[i] \approx y[i]=-b_{1} \cdot u[i-1]-b_{2} \cdot u[i-2]-\ldots-b_{p} \cdot u[i-p]
$$

Coefficients $b_{j}$ are calculated from the equation (8) for the minimum value of $J$ :

$$
J=\sum_{i=p+1}^{N}\left(u[i]+b_{1} \cdot u[i-1]+\ldots+b_{p} \cdot u[i-p]\right)^{2}
$$

where

$t_{s} N$ - time of one cycle of the separation process in a jig.

The results of filtration of the output signal from the counter (using period $t_{s}=3 \mathrm{~ms}$ ) are shown in Figure 3.

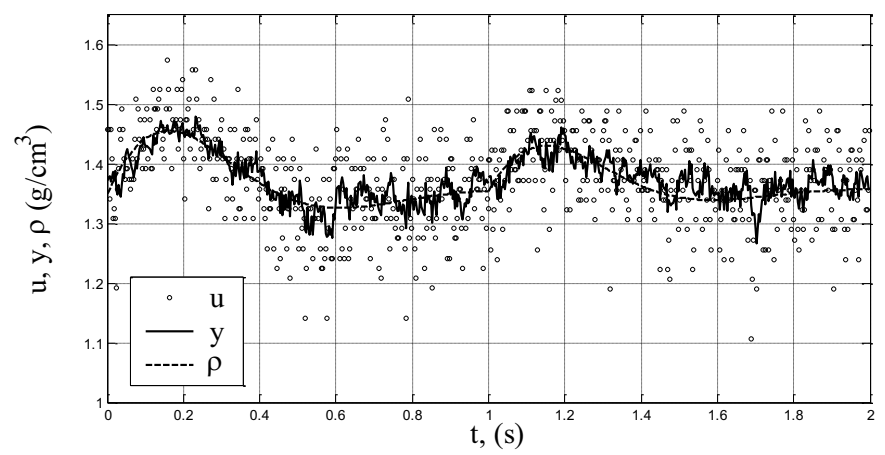

Figure 3. Output signal from the radiometric density meter after filtering in the predictive filter - two subsequent cycles of air/water pulsation.

\section{Field tests}

The radiometric density meter, presented in Fig. 2, was used to monitor changes in the jig's bed density during each cycle of air/water pulsations. The typical changes in the intensity of registered pulses from the detector and corresponding changes in the bed density, during one cycle of a jig operation and at the chosen bed level, are shown in Fig. 4. During the first $30 \mathrm{~ms}$ the bed is lifted by the water when the inlet air valve is opened, then follows the process of material loosening and finally the bed reaches its compressed state. The dynamic change in coal bed density depends on the point of density registration (position of the density measuring head), coal bed thickness, bed density distribution along the bed height and on parameters of the air pulsation cycle (pressure, time periods of air valves opening and closing).

The distribution of bed density was monitored for various positions (from -30 to $+15 \mathrm{~cm}$ ) of the measuring head, showed in Fig. 5a, above the bottom product discharge gate. The position " 0 " cm was accepted for the upper edge of the concentrate discharge gate. The thickness of the bed layer monitored in each point by the measuring head was ca. $3 \mathrm{~cm}$ and the length $-30 \mathrm{~cm}$. Density of the bed was determined in compressed states of the material. The distribution of the bed density is presented in Fig. 5 b.

The bed density in the compressed state varies from $1.0 \mathrm{~g} / \mathrm{cm}^{3}$ (water) at $+15 \mathrm{~cm}$ above the edge of the upper gate to $1.35 \mathrm{~g} / \mathrm{cm}^{3}$ at $-4 \mathrm{~cm}$ and to $1.45 \mathrm{~g} / \mathrm{cm}^{3}$ at $-20 \mathrm{~cm}$. The density at the position $-4 \mathrm{~cm}$ can be accepted as the density of the separation layer which reports in half to concentrate and in half to the refuse as the amplitude of bed pulsations during the measurements was ca. $3-4 \mathrm{~cm}$. 


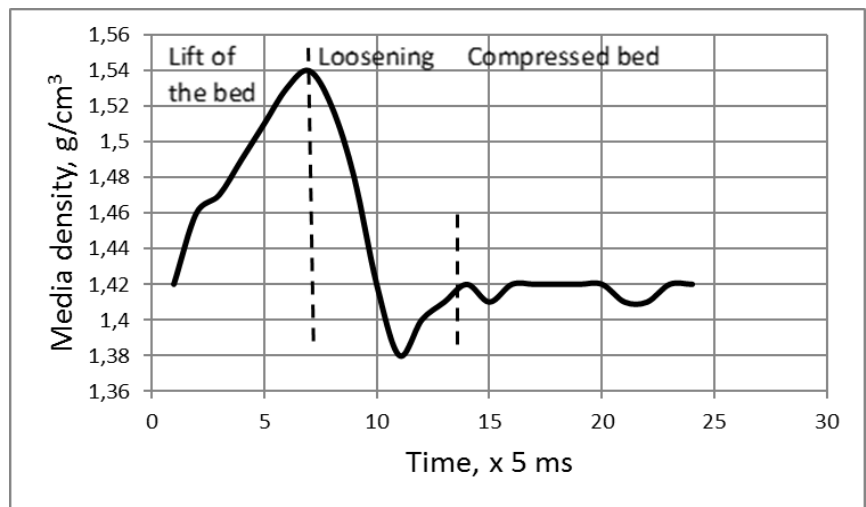

Figure 4. Typical change in the bed density during one cycle of pulsations (1.2 s) at the upper product split gate.

a)

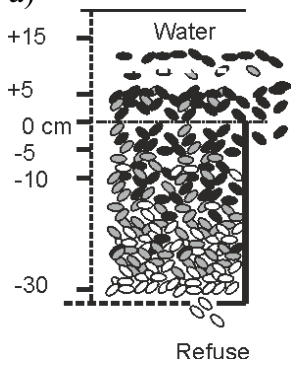

b)

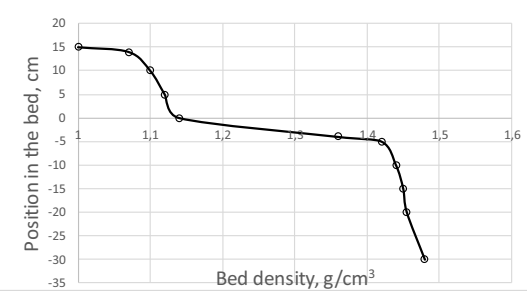

c)

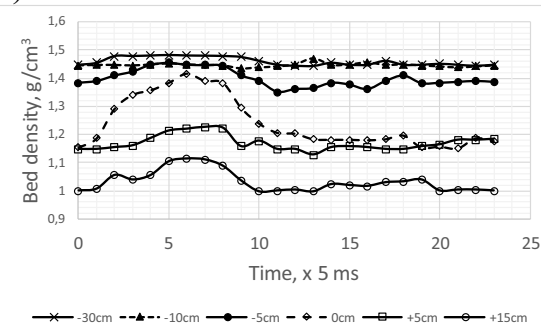

Figure 5. a) positions of measuring head during test; b) distribution of the bed density along its height; c) changes in the bed density over the one cycle of pulsations at various positions of measuring head.

The density of the dry material $\rho_{m}$ can be estimated from the equation (9) for a given density of the medium $\rho_{o}$ :

$$
\rho_{m}=\rho_{o}+\frac{\left(\rho_{o}-1\right) \cdot V_{H_{2} O}}{V_{z}}
$$

where:

$V_{H 2 O}$ - volume of water in a unit volume of medium,

$V_{z}$ - volume of dry material.

The ratio $V_{H 2 O} / V_{z}$ during the test was ca. 0.65 , hence the separation density (dry material) was ca. $1.58 \mathrm{~g} / \mathrm{cm}^{3}$.

Changes in the bed density over the one cycle of pulsations at various positions of the measuring head are shown in Fig. 5c. The density of the bed in lower layers is fairly constant (from $-30 \mathrm{~cm}$ to $-5 \mathrm{~cm}$ ) and for this reason changes in density when the bed is lifted are small. They become similar to Fig. 5 when loosening and compressing of the bed appears. Changes in the bed density over a cycle of pulsation cannot be a measure of the degree of material loosening but the period of time when material is in the loose state can be estimated. This information could be used for adjusting operation of air valves to stabilize the process of grains stratification.

Pulsations of the float (constant density of the float) during the increase of the feed tonnage are presented in Figure 6. The height of the float was $34 \mathrm{~cm}$, its " 0 " position corresponded to the position of the float bottom at " $-17 \mathrm{~cm}$ ". Full range of the float position was $20 \mathrm{~cm}$. The amplitude of pulsations varied from $3.5 \mathrm{~cm}$ up to $4.5 \mathrm{~cm}$. 
Changes in the bed density corresponding to the float pulsations are shown in Fig. 7. Initially, when the thickness of the bed is smaller, significant changes in density occur. The material at the height " $-4 \mathrm{~cm}$ " is lifted, then loosens and does not reach the compressed state. An optimal shape of density changes, when material loosens and reaches the compressed state can be observed for the position of the bottom of the float from $10 \%$ to $20 \%$. This information can be used for the control of the refuse discharge to stabilize the separation density in the jig.

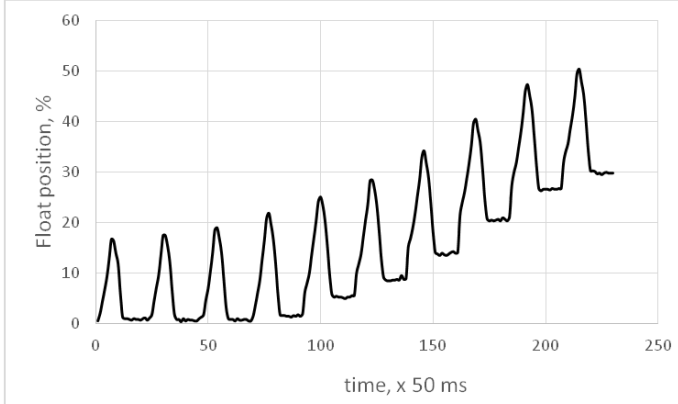

Figure 6. Pulsations of the float during the increase of the feed tonnage.

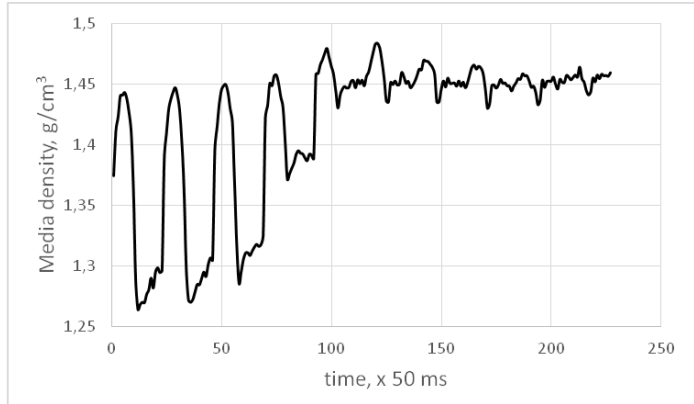

Figure 7. Changes in the bed density corresponding to changes in the float position (Fig. 6.) for $4 \mathrm{~cm}$ position of the measuring head.

\section{Conclusions}

The radiometric density meter can be effectively used for monitoring the distribution of density of the bed in a jig and for monitoring changes in density during a pulsation cycle. The signal from the meter contains significant stochastic noise due to random decay of the radiation source. For this reason it is necessary to apply a properly designed filter reducing the noise/signal ratio.

The change in the bed density during each cycle of pulsations, registered close to the edge of the upper product discharge gate, gives information on the bed lift, its loosening and compressing. The period of time of the bed loosening can be estimated from the shape of the signal and used for the control of operation of air valves.

Density of the bed in the compressed state indicates directly the density of the separation layer reporting in half to concentrate and in half to refuse. This signal can be used for better control of refuse discharge from the jig and better to stabilize the desired separation density.

\section{References}

1. J.W. Leonard, Coal Preparation (Society of Mining, Metallurgy, and Exploration, 1991)

2. R.P. King, Modeling and simulation of mineral processing systems (University of Utah, 2001).

3. G.J. Lyman, Coal Preparation, (1992).

4. R. Srinivasan, B.K. Mishra, S.P. Mehrotra, Coal Preparation, 20, 145 (1999).

5. Y. Xia, F.F., E. Wolfe, Int. J. Miner. Procces.,82(3), 164 (2007)

6. S. Cierpisz, in Proccedings of XVI International Coal Preparation Congress, (2013)

7. D. Bartelt, in Proccedings of IVth International Coal Preparation Congress, (1962)

8. G.J. Lyman, A. Jonkers, Gluckauf-Forschungshefte, 53, 131 (1992)

9. S. Cierpisz, J. Joostberens, Measurement, 88, 147 (2016)

10. S. Cierpisz, J. Joostberens, in Proccedings of Conference EMTECH 2016, Poland, (2016). 